Research Article

\title{
Noise Source Identification Method for a Carpet Tufting Machine Based on CEEMDAN-AIC
}

\author{
Wang Haohui (iD, Sheng Xiaowei, and Xu Yang \\ College of Mechanical Engineering, Donghua University, Shanghai 201620, China \\ Correspondence should be addressed to Wang Haohui; 1169165@mail.dhu.edu.cn
}

Received 2 February 2021; Revised 24 August 2021; Accepted 1 September 2021; Published 27 September 2021

Academic Editor: Wei He

Copyright (C) 2021 Wang Haohui et al. This is an open access article distributed under the Creative Commons Attribution License, which permits unrestricted use, distribution, and reproduction in any medium, provided the original work is properly cited.

In recent years, the noise reduction research of the carpet tufting machine has been developing slowly. The research gaps of the existing work mainly focus on the noise source identification for the carpet tufting machine. MEEMD (EEMD) has been proposed to apply to source recognition on textile machinery. Due to the uniqueness of the MEEMD/EEMD, it is difficult to set suitable white noise control parameters. MEEMD (EEMD) has only been tested via simulation; however, it has not been mathematically proven or evaluated. This leads to inevitable flaws in the research conclusions, and even some conclusions are wrong. The contribution of this paper is twofold. First, in order to recognize the noise source of a carpet tufting machine, a method based on complete ensemble empirical mode decomposition (CEEMDAN) and Akaike information criterion (AIC) is proposed. The CEEMDAN-AIC method is applied to measure the noise signal of a carpet tufting machine and analyzed every single effective component selected. Noise source identification is realized by combining the vibration signal characteristics of the main parts of the carpet tufting machine. CEEMDAN is used to decompose the measured noise signal of the carpet tufting machine into a finite number of intrinsic mode functions (IMFs). Then, singular value decomposition (SVD) is performed on the covariance matrix of the IMF matrix to obtain the eigenvalue. Next, the number of effective IMFs is estimated based on the AIC criterion, and the effective IMFs are selected by combining the energy characteristic index and the Pearson correlation coefficient method. Furthermore, reconstruction and comparison of the decomposed signals of MEEMD and CEEMDAN proved that CEEMDAN is effective and accurate in source recognition. The results show that the noise signal of the carpet tufting machine is a mixture of multiple noise source signals. The main noise sources of the carpet tufting machine include shock caused by the impact of the tufted needle and looped hook and vibration of the hook-driven shaft and pressure plate. It provides theoretical support for the noise reduction of the carpet tufting machine.

\section{Introduction}

ANSI [1] stipulates that, for single-level noise, continuous noise exposure shall not exceed $80 \mathrm{~dB}$ when working for more than 8 hours. In 2013, China's newly revised GB/T 50087-2013 [2] stipulates that the noise of the workshop is $85 \mathrm{~dB}$. At present, the noise of the textile workshop is generally above $85 \mathrm{~dB}$. Even with improvement of the characteristics of the carpet tufting machine, such as making it wide, heavy, high speed, and complex in the mechanism, the noise generated is greater. Therefore, research on the noise reduction of textile machinery is urgent and significant.
Generally speaking, the structure of the carpet tufting machine is as complex as its transmission path. The weaving process includes high-speed rotation, reciprocating, multimotion coupling, impact, friction, and other conditions. It leads to more than one noise source. Even the same sound source often has multiple parts that produce sound, and the noise condition is unusual and complex [3-5]. Therefore, it is necessary to understand the characteristics of each sound source and the weight of its total noise. Then, locate and identify the main noise sources in order to formulate reasonable noise reduction measures for a carpet tufting machine with multiple noise sources. 
Noise energy is usually concentrated in the low-frequency band. Noise source feature extraction is one of the key techniques for source identification, especially for the carpet tufting machine. Therefore, how to accurately extract the source feature from complex noise is always a difficult problem in the textile industry. Some research studies use classical Fourier analysis and wavelet transform as the basis of noise signal processing [6-8]. The Fourier analysis cannot express the time-frequency local performance of the signal. The results cannot reflect the real features for the target signal well $[9,10]$. Wavelet transform can multiscale refine the signal by calculating flex and transition. It can solve the problem that the size of the Fourier transform window cannot change with frequency. However, wavelet transform is still based on Fourier analysis and limited by the selection of the wavelet basis function and decomposition layer. For carpet tufting machine noise signal processing, we hope that we can not only get the frequency information of the signal but also get the law of the frequency changing with time. As an empirical signal analysis method, empirical mode decomposition (EMD) overcomes the limitation of Fourier transform fundamentally and can theoretically decompose any signal into IMFs [11-14]. The major drawback of the EMD is the frequent appearance of mode mixing. Some experts have put forward improved EMD algorithms to solve mode mixing of EMD. Among them, the more universal and effective algorithms are ensemble EMD (EEMD) $[15,16]$. Although EEMD reduces mode mixing, it has greater error between the original signal and the reconstruction signal. For textile machinery, Xu et al. $[3,4]$ proposed modified ensemble empirical mode decomposition (MEEMD). Although MEEMD reduces the reconstruction error to a certain extent, it still cannot meet accuracy requirements. Marcelo and Gastón [17] presented complete ensemble EMD with adaptive noise (CEEMDAN). It can solve mode mixing and reduce calculation with negligible reconstruction error [18-20].

In this paper, the decomposition results of CEEMDAN and MEEMD are compared. CEEMDAN has higher accuracy, and it is more suitable for the noise source extraction for the textile industry. The CEEMDAN algorithm is combined with the Akaike information criterion (AIC) source number estimation method, and the CEEMDANAIC method is for the noise source identification of the carpet tufting machine. Then, the CEEMDAN-AIC method is applied for the identification of the noise source of a carpet tufting machine, and its main noise source is accurately identified. This can provide theoretical support for the active noise reduction of the carpet tufting machine.

\section{Noise Characteristic Analysis of a Carpet Tufting Machine}

2.1. Structure of the Carpet Tufting Machine. In this paper, the experiment object is a four-meter tufted carpet tufting machine. It is shown in Figure 1. The carpet tufting machine is mainly composed of four parts: host system, loop-forming system, yarn feeding system, and needle bed and traverse system. The host system is used to make the rotary motion of

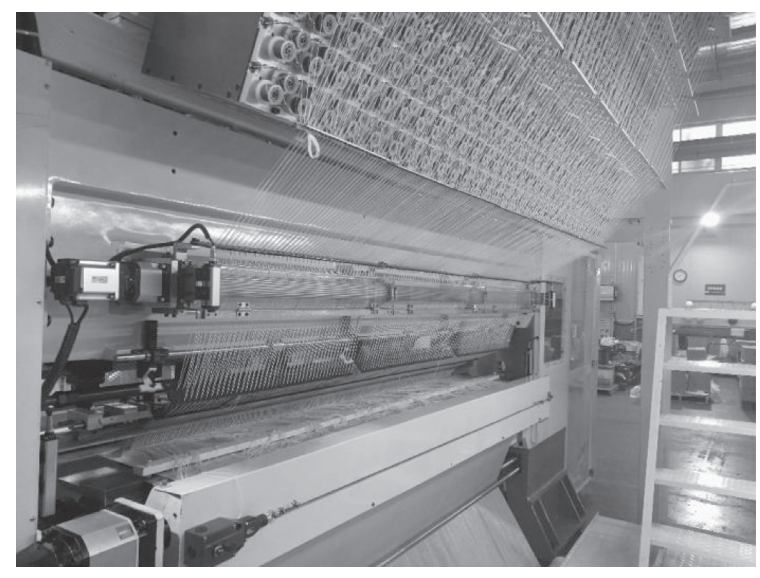

FIgURE 1: The carpet tufting machine.

the spindle transformed into the loop/cutting motion of the loop hook swinging left and right and the flocking motion of the tufting needle up and down. The loop-forming system is used to complete the loop-forming movement of the carpet tufting machine. The yarn feeding system is used to send yarn to the loop forming. The needle bed and traverse system is used to put yarns of different colors inserted into the tufted pinholes at intervals. Assist the loop-forming system in completing the fabric weaving. Under the operating condition that the main shaft speed of the carpet tufting machine is $360 \mathrm{rpm}$, LV-FS01 and Quick Signal Analyzer (Quick SA) real-time signal analysis software are used to collect the vibration signals of the main vibration parts of the carpet tufting machine, and the collected vibration signals are analyzed one by one. The main vibration frequencies of the parts are shown in Table 1. The sampling frequency is set to $2048 \mathrm{~Hz}$, and the sampling time is $20 \mathrm{~s}$.

2.2. The Acquisition and Preprocessing of Noise Signals. Under the same conditions, the noise signals of the carpet tufting machine near the workers' ears are collected. According to GB/T 7111.6-2002 "Textile machinery-Noise test code-Part 6: Fabric manufacturing machinery," the sound pressure sensor is arranged at a distance of $1 \mathrm{~m}$ from the machine surface and at a height of $1.6 \mathrm{~m}$ from the table position.

The noise signals are collected in this experiment by using a sound pressure sensor BK4961 combined with the DHAS dynamic signal analysis system.

The sampling frequency is $8192 \mathrm{~Hz}$, and the sampling time is $20 \mathrm{~s}$. A total of 6 experiments are carried out, and the experimental site layout is shown in Figure 2.

All the noise signals collected are analyzed preliminarily. In order to improve the computational efficiency, a typical data length of $1 \mathrm{~s}$ is selected as the analysis object.

The signal waveform and spectrum obtained after the fast Fourier transform of the signal are shown in Figure 3. It is seen in the figure that the frequency of the noise signals is mainly distributed below $400 \mathrm{~Hz}$. And the frequency component is complex, mainly composed of low-frequency noise within $0-300 \mathrm{~Hz}$. 
TABLE 1: The main vibration frequencies of the parts.

\begin{tabular}{lc}
\hline Part & Main vibration frequency $(\mathrm{Hz})$ \\
\hline Pressure plate & 5,52 \\
Motor spindle & 6,47 \\
The hook & 5 \\
The hook-driven shaft & 5,57 \\
The hook main shaft & 5 \\
Machine base & $5,26,37$ \\
\hline
\end{tabular}

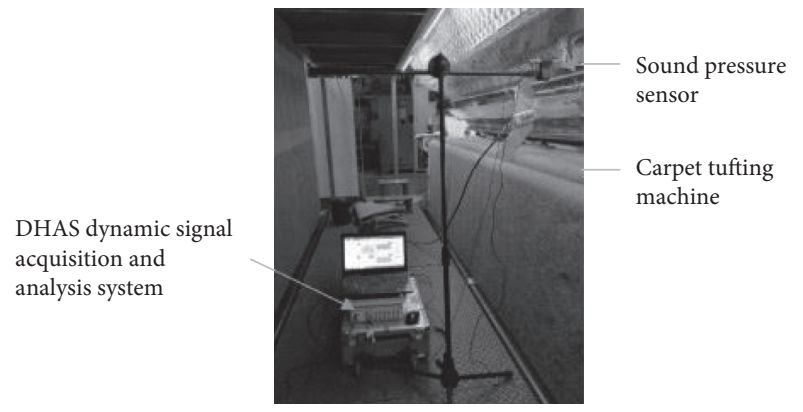

Figure 2: Experimental arrangement.

An appropriate window function is used for short-time Fourier transform of the noise signal near the workers' ears. It is clear from the time-frequency diagram shown in Figure 4 that the noise source is not unique. The signal characteristics of each frequency band are shown in Table 2.

\section{CEEMDAN-AIC Noise Recognition Algorithm}

The core of the CEEMDAN-AIC approach is the CEEMDAN algorithm and AIC guidelines. The CEEMDAN-AIC algorithm flowchart is shown in Figure 5.

(1) CEEMDAN decomposition of the signal: CEEMDAN decomposition of the single-channel observation signal yields a finite number of IMF components.

(a) Add two sets of positive and negative white noise signals of equal absolute value to the signal to be decomposed $x(t)$ to form a new signal $x^{i}(t)$. Multiple first-order components $\mathrm{IMF}_{1}^{i}$ are obtained by EMD of the__new signal. The first-order final component $\mathrm{IMF}_{1}$ is obtained by averaging several first-order components. The first residue can be expressed as

$$
r_{1}(t)=x(t)-\overline{\mathrm{IMF}_{1}} .
$$

(b) Add two sets of positive and negative white noise signals of equal absolute value to $r_{1}(t)$ to form a new signal $r_{1}^{i}(t)$. Multiple second-order components $\mathrm{IMF}_{2}^{i}$ are obtained by EMD of the signal. The second-order final component $\mathrm{IMF}_{2}$ is obtained by averaging several second-order components. The second residue can be expressed as

$$
r_{2}(t)=r_{1}(t)-\overline{\mathrm{IMF}_{2}} \text {. }
$$

(c) For $k=1,2,3, \Lambda$, go to Step b for next $k$. The $k$ th residue can be expressed as

$$
r_{k}(t)=r_{k-1}(t)-\overline{\mathrm{IMF}_{k}} \text {. }
$$

Finally, CEEMDAN can be expressed as

$$
x(t) \longrightarrow \text { CEEMDAN } \sum_{i=1}^{k} \overline{\mathrm{IMF}_{i}}+r_{k}(t),
$$

where $x(t)$ is the observation signal.

(2) Estimate effective IMFs:

The IMF matrix is obtained after the signal is decomposed by CEEMDAN. The IMF matrix's covariance matrix is decomposed by SVD. Multiple eigenvalues corresponding to IMFs can be obtained. The noise background of the carpet tufting machine is colored noise, and the accuracy of the AIC for estimating the number of sources in the background of colored noise is poor. Therefore, $\mathrm{Xu}$ et al. [3] smoothed out the noise eigenvalues by diagonally loading the covariance matrix in order to make it to be applied to the background of colored noise. The corrected eigenvalue $\mu_{i}=\lambda_{i}+\lambda_{D L}, \quad i=1,2, \Lambda, m$. $\lambda_{D L}=\sqrt{\sum_{i=1}^{m} \lambda_{i}}$. The correction can be expressed as

$$
\begin{aligned}
\mathrm{AIC}= & -2 N(m-L) \lg \left(\frac{\prod_{i=L+1}^{m} \mu_{i}^{(1 / m-L)}}{(1 / m-L) \sum_{i=L+1}^{m} \mu_{i}}\right) \\
& +2 L(2 m-L),
\end{aligned}
$$

where $N$ is the number of samples and $L=1,2, \Lambda, m-1$. The AIC value of $L$ is calculated from 1 to $m-1$. $L$ corresponding to the smallest AIC value is the number of effective components. Combining the energy characteristic index with the Pearson correlation coefficient [3], the total energy of each IMF and the correlation coefficient between each IMF and the original signal are calculated. According to the correlation coefficient of each IMF, all the IMFs are reordered to find out the most significant components.

(3) Noise source identification:

The signal characteristics of the effective IMFs are analyzed to complete the noise source identification.

\section{Noise Source Identification of a Carpet Tufting Machine}

CEEMDAN decomposition of the noise signal is done. The amplitude of the added white noise is 0.3 times of the RMS of the noise signal. The number of added white noise is 200 . 


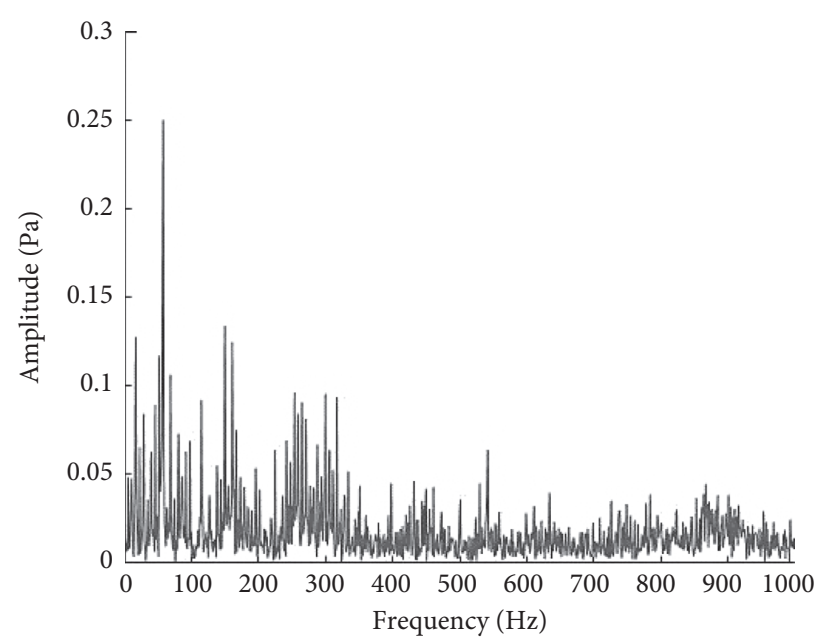

(a)

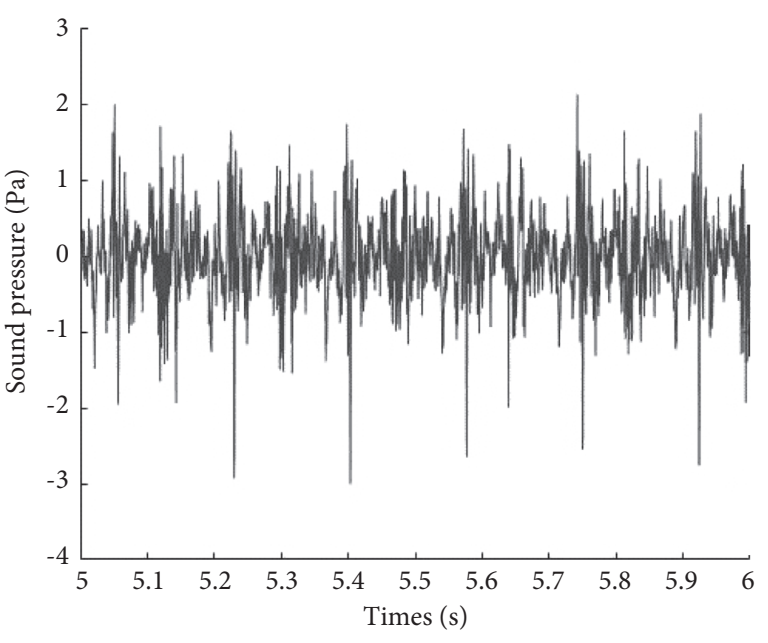

(b)

FIGURE 3: The pretreatment of the noise signal. (a) Amplitude-frequency of the noise signal. (b) Amplitude-time of the noise signal.

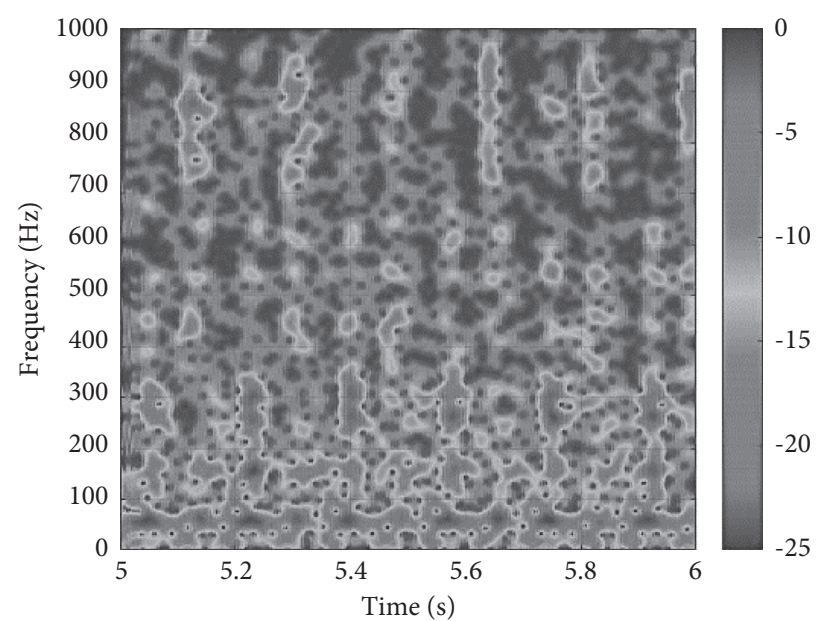

FIGURE 4: Time-frequency of the noise signal.

TABLE 2: The signal characteristics of each frequency band.

\begin{tabular}{lc}
\hline Frequency band $(\mathrm{Hz})$ & Signal characteristics \\
\hline $700-900$ & Showing a certain periodicity \\
$250-350$ & Showing a certain periodicity with the spindle rotation cycle \\
150 or so & The signal did not change significantly over time, time-invariant stable signal \\
$0-60$ & Showing a certain periodicity \\
\hline
\end{tabular}

Seven IMFs are obtained. The decomposition result is shown in Figure 6.

\subsection{Estimation of Effective IMFs of Carpet Tufting Machine} Noise. The covariance matrix of the IMFs is calculated and then decomposed by SVD. The eigenvalues were obtained: $0.0857,0.0551,0.0318,0.0198,0.0083,0.0015$, and 0.0002 . The AIC after correction is shown in Figure 7. The smallest AIC corresponds to $L=4$. Then, the estimated number of valid IMFs is 4.
Combining the energy characteristic index with the Pearson correlation coefficient, the total energy of each IMF and the correlation coefficient between each IMF and the original signal are calculated. The results are shown in Table 3.

It can be seen from Table 3 that the energy and correlation coefficients of IMF1-IMF4 are large. Because the number of effective IMFs of the carpet tufting machine decomposed by CEEMDAN is 4, IMF1-IMF4 are effective. IMF5-IMF7 are not effective as their energy and correlation coefficient are small. 


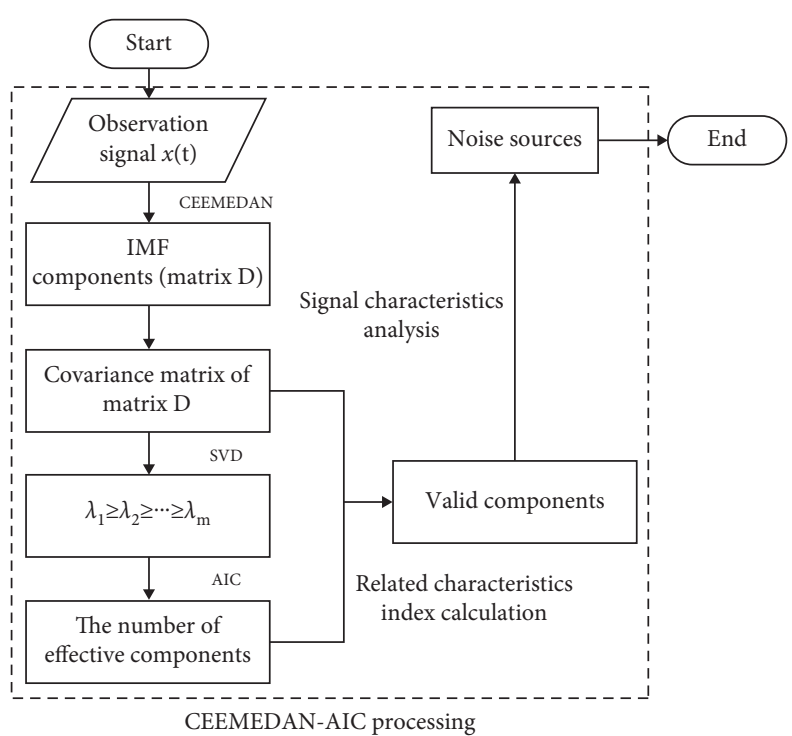

Figure 5: Flowchart of the CEEMDAN-AIC algorithm.


FIgure 6: Results of CEEMDAN decomposition.

4.2. Characteristic Analysis of Effective Noise and Noise Source Identification. Each amplitude-frequency figure of each effective IMF of carpet tufting machine noise shown in Figure 8 is obtained through the fast Fourier transform. Figure 9 shows the time-frequency of IMF1-IMF4.

According to Figure 6 and Figure 9(a), it can be seen that IMF1 is a shock signal. It impacts about 6 times per second. This corresponds to the spindle motor speed $(360 \mathrm{r} / \mathrm{min})$. When the spindle motor is working, it rotates once in $0.2 \mathrm{~s}$. At this time, the needle row punctures the base cloth every $0.2 \mathrm{~s}$. The peak value appears on the amplitude-time figure of IMF1 every $0.2 \mathrm{~s}$ (Figure 6). Therefore, IMF1 is the shock noise caused by the impact of the tufted needle and loop hook.

Background noise signals in the factory are collected and decomposed by CEEMDAN. The time-frequency of the background noise is shown in Figure 10.

Comparing Figure 10 with Figure 9(b), it can be seen that the frequency distribution is similar and that all signal frequency characteristics change with time. Therefore, IMF2 is derived from the background noise.

The vibration time-frequency of the hook-driven shaft and pressure plate is shown in Figure 11. The amplitudefrequency of IMF3 and IMF4 is extracted from Figure 8 and 


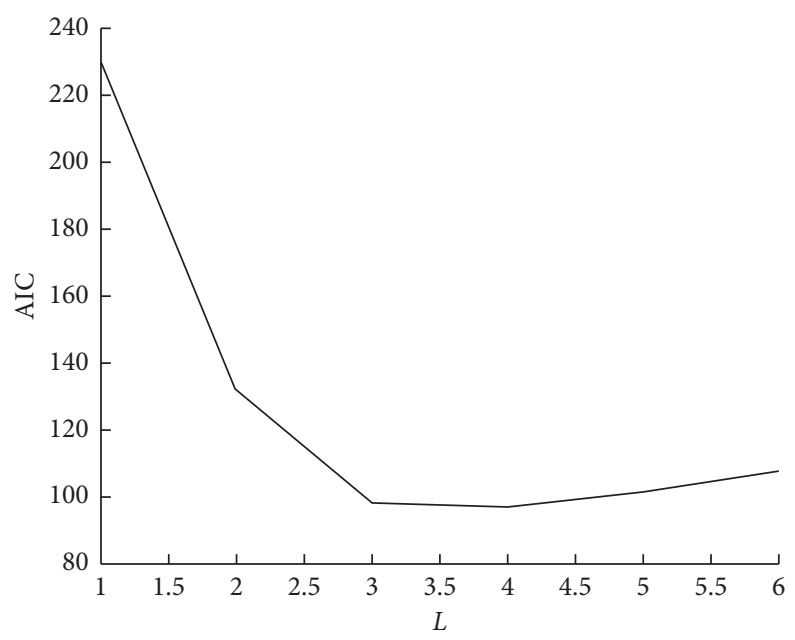

Figure 7: Calculation results of the AIC.

TABLE 3: The total energy of each IMF and the correlation coefficient.

\begin{tabular}{|c|c|c|c|c|c|c|c|}
\hline & IMF1 & IMF2 & IMF3 & IMF4 & IMF5 & IMF6 & IMF7 \\
\hline Energy $\left(\mathrm{Pa}^{2}\right)$ & 148.599 & 108.451 & 69.736 & 175.160 & 40.515 & 17.224 & 3.16 \\
\hline Correlation coefficient & 0.568 & 0.560 & 0.420 & 0.516 & 0.213 & 0.130 & 0.055 \\
\hline
\end{tabular}
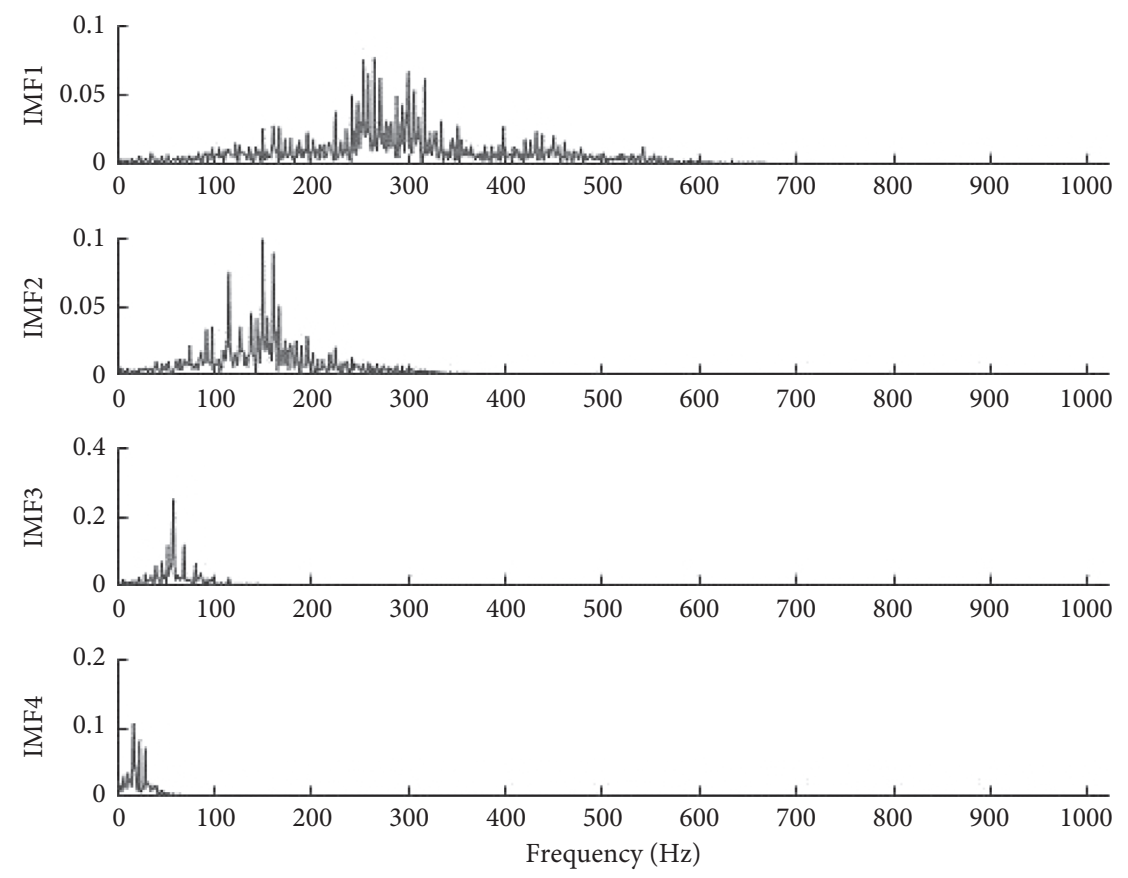

FIgURE 8: Amplitude-frequency of IMF1-IMF4.

amplified in the frequency axis, resulting in Figure 12. According to Figure 12(a), an obvious peak is at $58 \mathrm{~Hz}$, the frequency does not change with time, and no periodic shock characteristics and concentrated frequency distribution were observed. The amplitude of $58 \mathrm{~Hz}$ is the largest in the whole frequency domain, and it is about 10 times of the spindle rotation frequency. Figure 9(c) and Figure 11 show that the phase difference between the pressure plate and the hook- driven shaft is about $180^{\circ}$ and alternates with each other on the whole time axis. By measuring the main vibration of the hook-driven shaft and pressure plate $(58.3 \mathrm{~Hz}, 52.7 \mathrm{~Hz})$, it can be known that IMF3 is the vibration noise caused by the vibration of the hook-driven shaft and pressure plate.

Figure 12(b) shows an obvious peak at $17 \mathrm{~Hz}$. The distribution of frequency is narrow and below $20 \mathrm{~Hz}$. The audible frequency range of the human ear is from $20 \mathrm{~Hz}$ to 


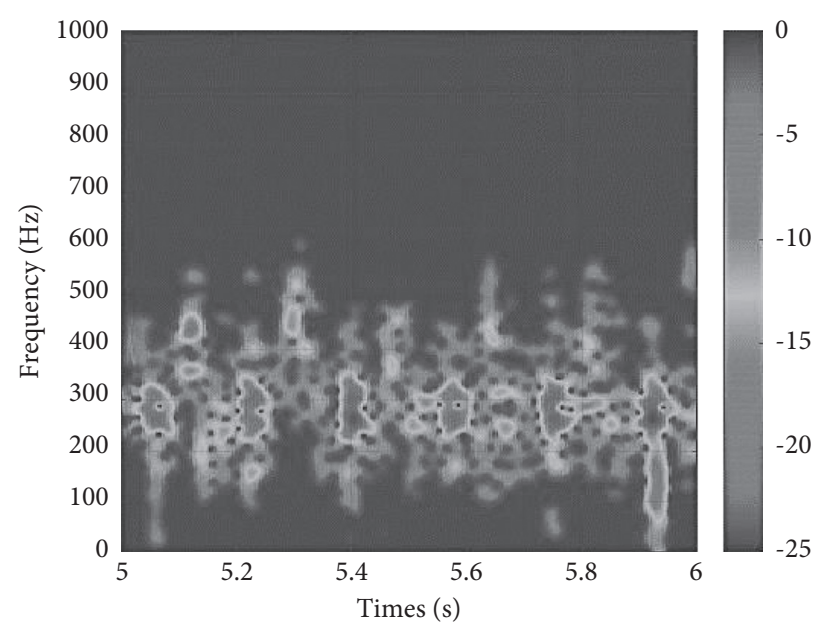

(a)

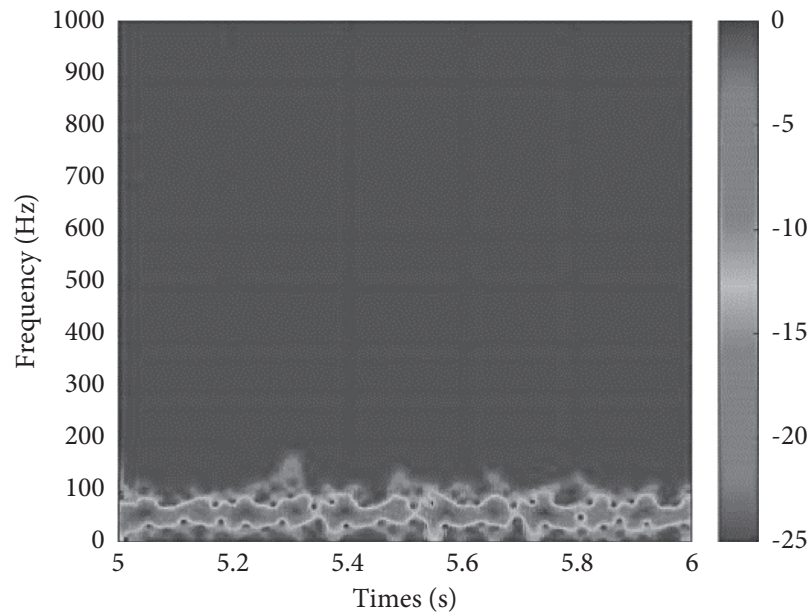

(c)



(b)

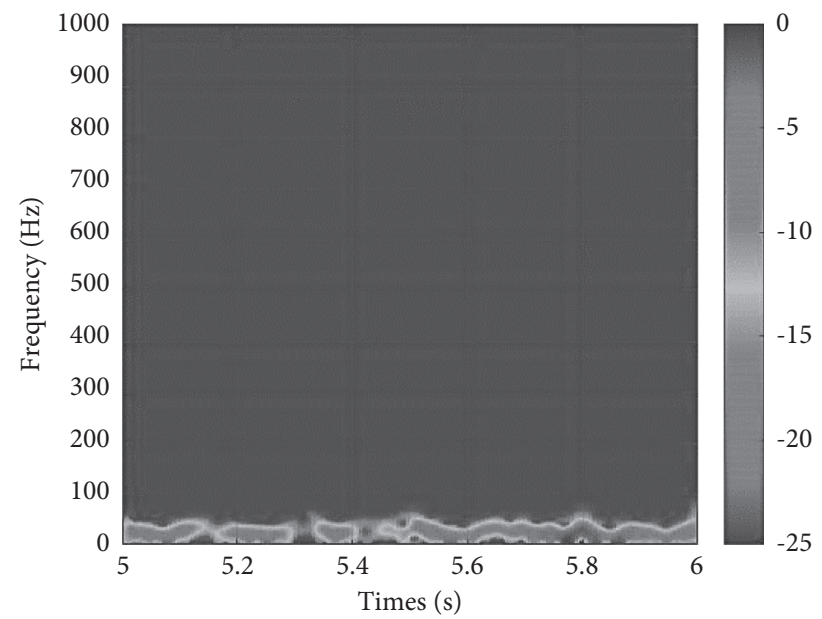

(d)

Figure 9: (a) Time-frequency of IMF1. (b) Time-frequency of IMF2. (c) Time-frequency of IMF3. (d) Time-frequency of IMF4.

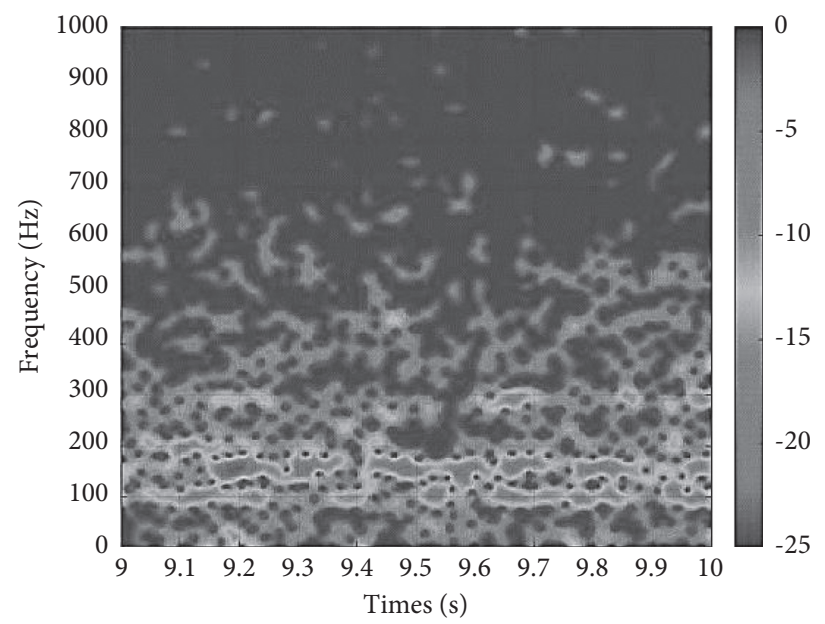

FIgURE 10: Time-frequency of the background noise. 


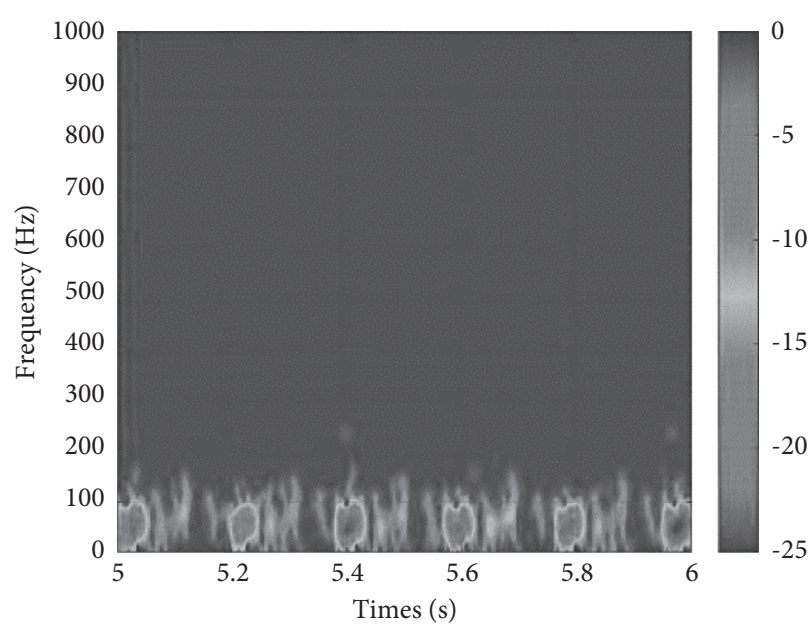

(a)

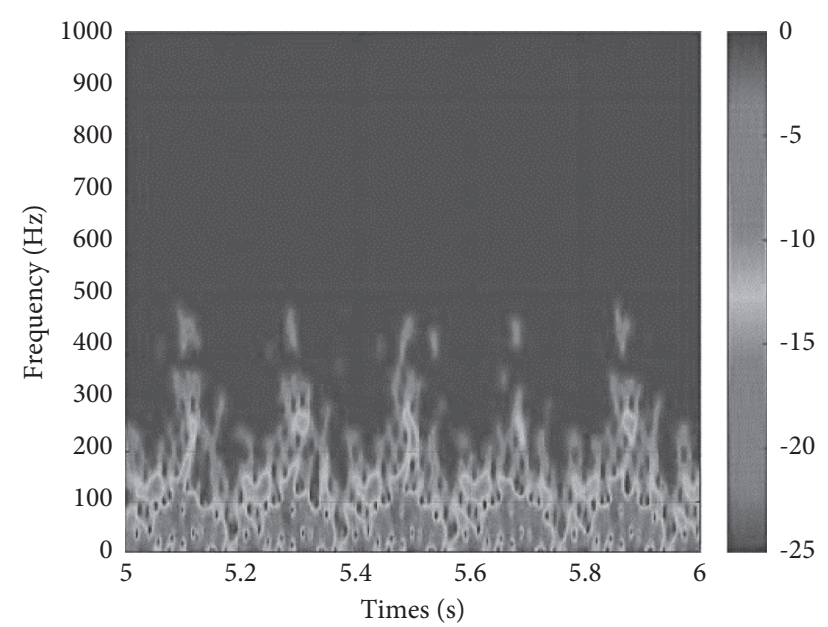

(b)

Figure 11: Vibration time-frequency of the pressure plate and hook-driven shaft. (a) Time-frequency of the pressure plate. (b) Timefrequency of the hook-driven shaft.

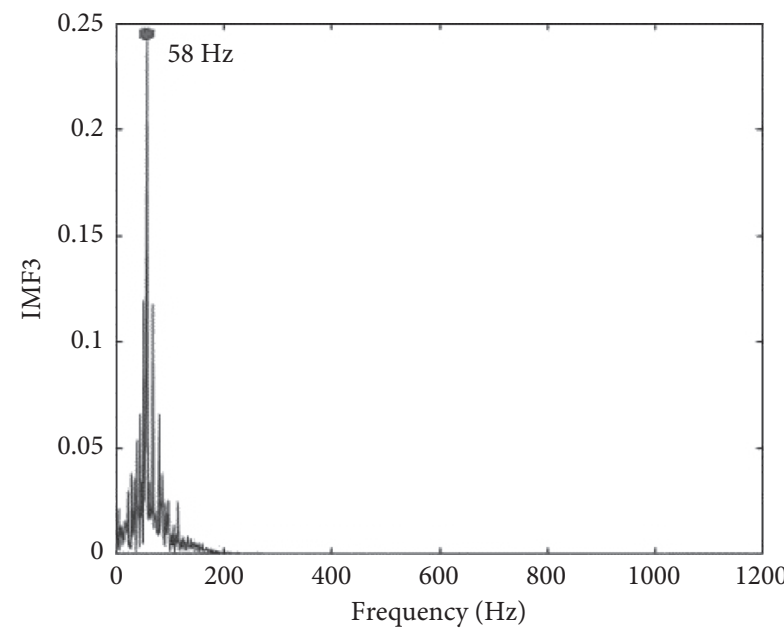

(a)

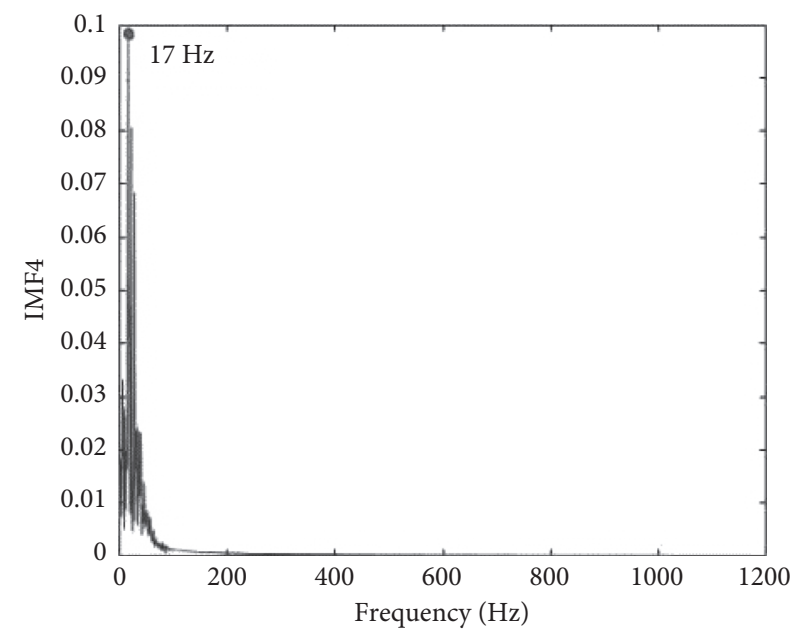

(b)

FIgURE 12: (a) Amplitude-frequency of IMF3. (b) Amplitude-frequency of IMF4.

$20 \mathrm{kHz}$. Therefore, IMF4 belongs to the infrasound wave, and it cannot be recognized by the human ear. It is not necessary for IMF4 to identify the noise source.

4.3. Comparing with the MEEMD Noise Recognition Algorithm. In Section 4.1, CEEMDAN-AIC is directly applied to decompose and analyze the noise signal, but this is not prudent enough to verify the accuracy of the method. It is necessary to add one accurate calculation example to verify the accuracy of the method, and the results of CEEMDAN and MEEMD should be compared.

According to the features of the measured noise signal, the simulation signal of the accurate calculation example can be expressed (Figure 13):

$$
\left\{\begin{array}{l}
m_{1}=5 e^{-(t-500 / 100)^{2} \pi} \cos \left(\frac{5 \pi}{6}(t-1000)\right) \\
m_{2}=\cos \left(\frac{4 \pi}{125}(t-1000)+10\left(\sin \left(\frac{\pi t}{2500}\right)-86\right)\right), \quad t \in[0,2000] . \\
m_{3}=2 e^{-\pi(t-1400 / 200)^{2}} \cos \left(7 \pi\left(\frac{t-1400}{128}\right)\right)
\end{array}\right.
$$



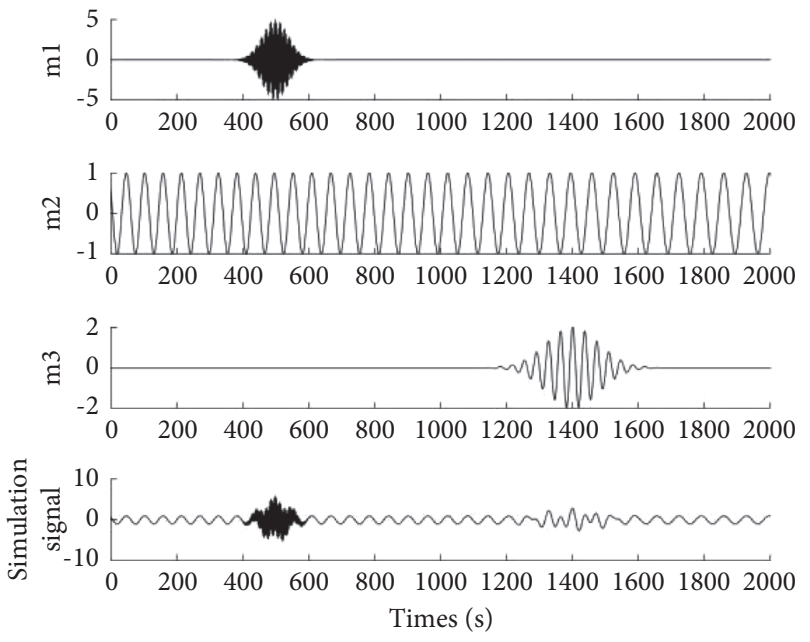

FIgURE 13: Simulation signal.

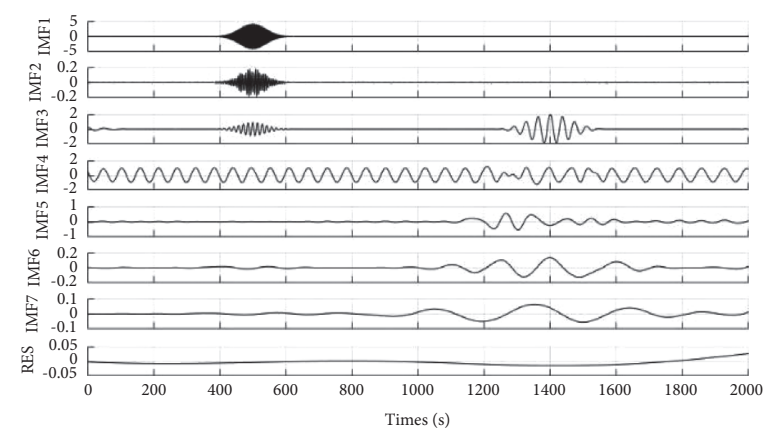

Figure 14: Decomposition of the simulation signal by MEEMD.

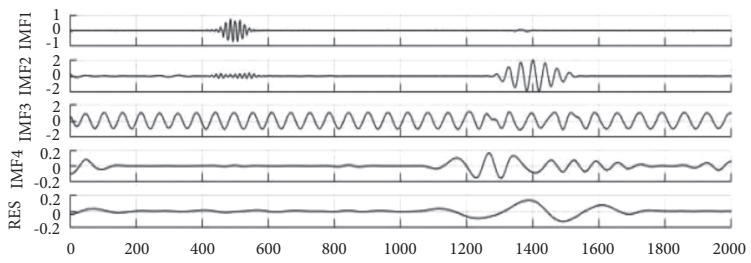

Figure 15: Decomposition of the simulation signal by CEEMDAN.

Simulation signal $=m_{1}+m_{2}+m_{3}$.

According to Figures $13-15$, it is obvious that MEEMD presents strong mode mixing and contains the spurious component. For MEEMD, IMF1-IMF4 have large energy, and they do not represent real information of the simulation signal. For CEEMDAN, the decompositions were stopped once the IMF was satisfied for the current residue. Figures 16 and 17 show that compared with MEEMD, the reconstructed signal error generated by CEEMDAN is negligible, and the energy distribution is more uniform. It can well decompose the simulation signal into three effective components. CEEMDAN can represent real information of the simulation signal more accurately than MEEMD.
MEEMD of the noise signal is shown in Figure 18. 10 IMFs are obtained. Comparing with Figure 6, it is obvious that MEEMD shows frequent appearance of mode mixing [4].

Each amplitude-frequency figure of each effective IMF of MEEMD shown in Figure 19 is obtained through the fast Fourier transform.

The energy error of the MEEMD algorithm is very large. It can change the energy distribution of the modal component. This will seriously affect the identification of the noise source. The conclusions in [4] show that the noise of the carpet tufting machine is mainly composed of friction between the tufting needle and base cloth (IMF1), the shock noise caused by the impact of the tufted needle and loop 




FIGURE 16: MEEMD reconstruction signal error of the simulation signal.



FIGURE 17: CEEMDAN reconstruction signal error of the simulation signal.

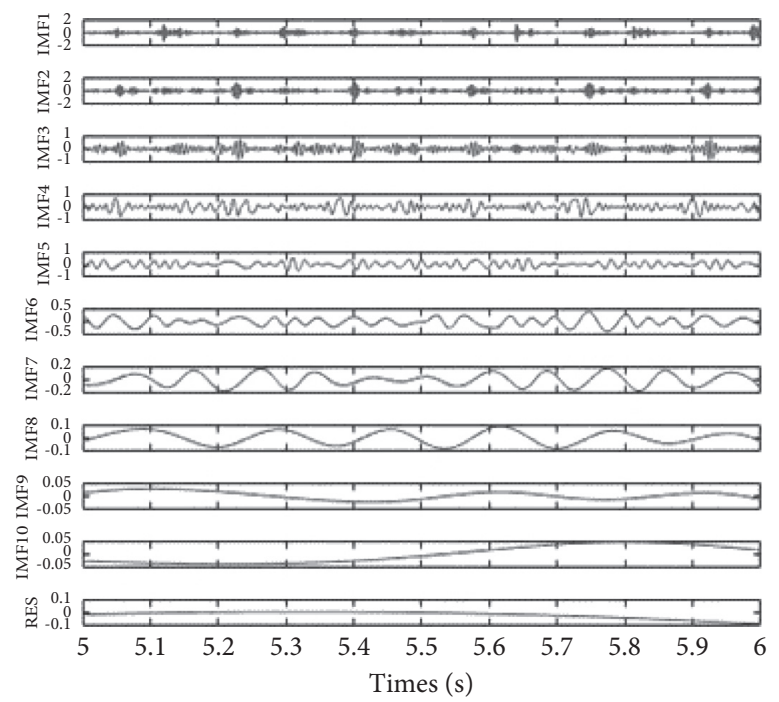

Figure 18: Results of MEEMD. 


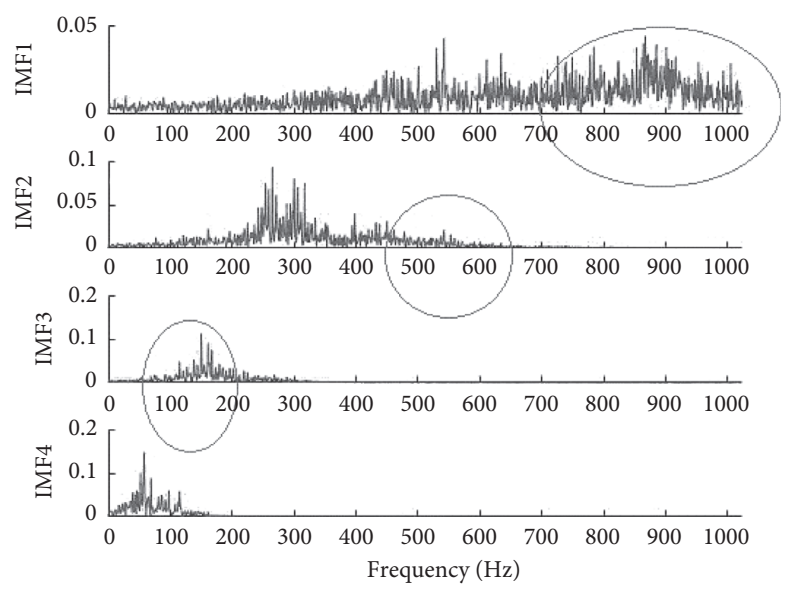

Figure 19: Amplitude-frequency of IMF1-IMF4.

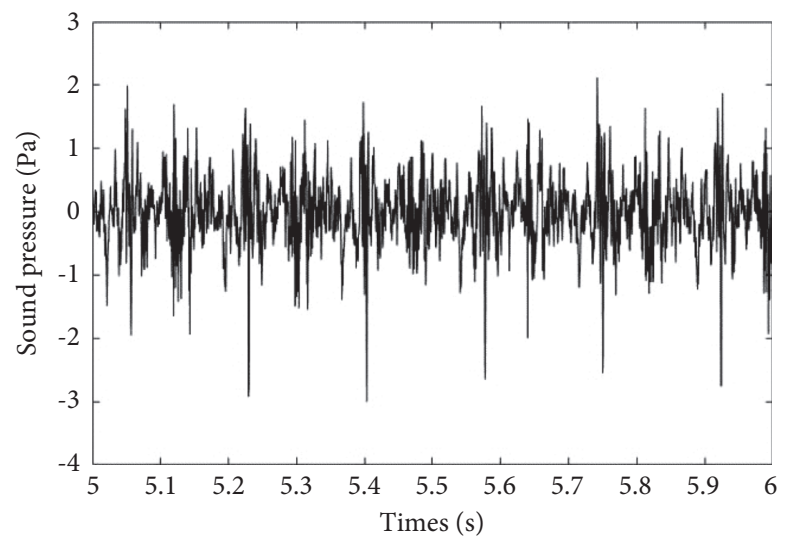

FIGURE 20: CEEMDAN reconstruction signal.

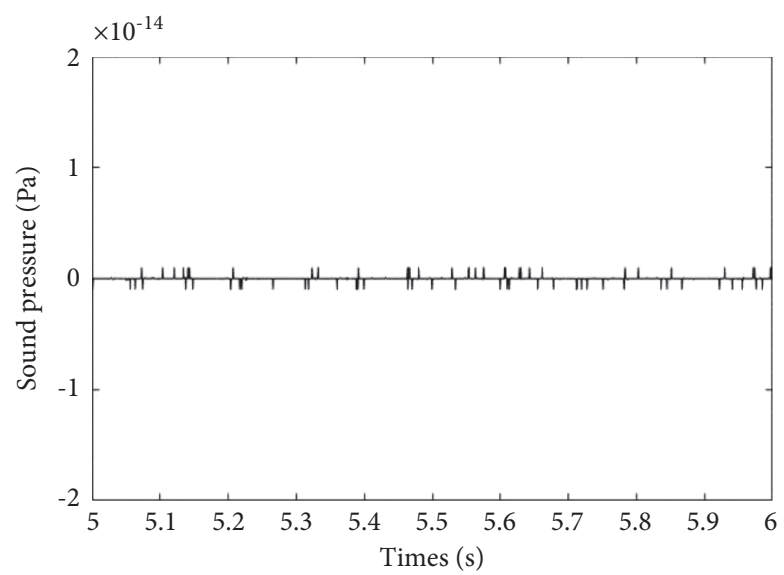

Figure 21: CEEMDAN reconstruction signal error.

hook (IMF2), background noise (IMF3), and vibration noise of the hook-driven shaft and pressure plate (IMF4). In fact, IMF1 is a spurious component caused by mode mixing. It is unable to find out the parts' vibration signal corresponding to IMF1. Most energy in IMF1 is provided by white noise. This means friction between the tufting needle and base cloth does not contribute to the noise signal. Due to the features of MEEMD/EEMD, it is difficult to remove the white noise from decomposition results. Noise source identification based on MEEMD is more relying on experience and more prone to error. CEEMDAN has high completeness. It can remove the white noise from 




FIgURE 22: MEEMD reconstruction signal.

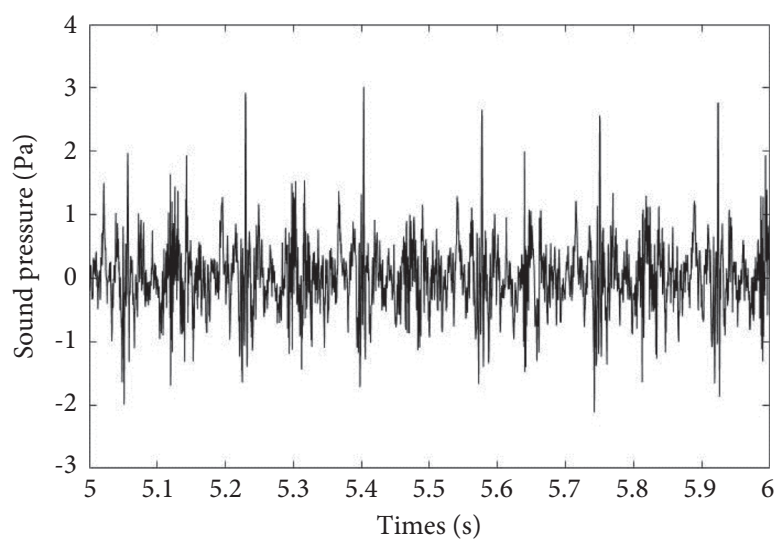

FIGURE 23: MEEMD reconstruction signal error.

TABLE 4: Energy of each signal.

\begin{tabular}{lc}
\hline Signal & Energy $\left(\mathrm{Pa}^{2}\right)$ \\
\hline Original noise signal & 630.1 \\
CEEMDAN reconstruction signal & 630.2 \\
MEEMD reconstruction signal & 2025 \\
\hline
\end{tabular}

decomposition results. This can reduce the appearance of the spurious component in decomposition results.

In order to illustrate this problem, the error of the reconstruction signal of CEEMDAN and MEEMD should be compared.

According to Figures 20-23 and Table 4, compared with the CEEMDN reconstruction signal, the energy error of the MEEMD reconstruction signal is $31.1 \%$. It is obvious that CEEMDAN is more suitable for the noise source extraction of the carpet tufting machine. Meanwhile, the conclusions [4] are not accurate.

\section{Conclusions}

In this paper, using the CMEEMDAN-AIC algorithm combined with the carpet tufting machine structure characteristics and related experimental analysis, the noise sources of the carpet tufting machine are identified, concluded as follows:

(1) A CEEMDAN-AIC algorithm which is applied to the noise source identification of the carpet tufting machine is presented.

(2) The CEEMDAN-AIC algorithm is used to deal with noise measured near the ear of workers. Four effective IMFs of the noise of the carpet tufting machine are obtained. Among them, IMF2 and IMF4 are, respectively, background noise and infrasound.

(3) Based on the analysis of IMF1 and IMF3, combined with the vibration characteristics of each machine part of the carpet tufting machine, it can be concluded that the noise is mainly composed of the vibration of the hook-driven shaft and pressure plate and the impact of the tufted needle and loop hook.

(4) Compared with other noise source extraction techniques (MEEMD), CEEMDAN can remove the white noise from decomposition results and reduce the appearance of the spurious component in decomposition results. CEEMDAN has better performance for actual carpet tufting machine noise signals. The error can be reduced by $31.1 \%$. 


\section{Data Availability}

The research data are from previously reported studies and datasets. The related research data can be made available from the corresponding author upon request.

\section{Conflicts of Interest}

The authors declare no conflicts of interest with respect to the research, authorship, and/or publication of this article.

\section{Acknowledgments}

This work was supported by the National Natural Science Foundation of China (51675094).

\section{References}

[1] American National Standards Institute, ANAI Guide, American National Standards Institute, New York, NY, USA, 1918.

[2] Ministry of Housing and Urban-Rural Development of the People's Republic of China, GB/T 50087-2013, Code for Design of Noise Control of Industrial Enterprises, China Building Industry Press, Beijing, China, 2014.

[3] Y. Xu, Z. Zhang, A. Li, and X. Sheng, "Noise source identification method for a warp machine based on MEEMD_AIC," Fibres and Textiles in Eastern Europe, vol. 28, no. 141, pp. 55-61, 2020.

[4] X. Zhang, "Noise source identification method of tufted carpet loom based on MEEMD-AIC," Journal of Vibration, vol. 28, 2018.

[5] H. Ling, Research on Noise Source Location of Tufted Carpet Loom Base on Transfer Path Analysis Method, Donghua University, Shanghai, China, 2018.

[6] H. Yang, Y. A. Li, and G. H. Li, "Noise reduction method of ship radiated noise with ensemble empirical mode decomposition of adaptive noise," Noise Control Engineering Journal, vol. 64, pp. 230-242, 2016.

[7] Y. Li, L. Wang, X. Li, and X. Yang, "A novel linear spectrum frequency feature extraction technique for warship radio noise based on complete ensemble empirical mode decomposition with adaptive noise, duffing chaotic oscillator, and weighted-permutation entropy," Entropy, vol. 21, no. 507, 2019.

[8] X. U. Yang, "Noise Sources identification of carpet tufting machine based on empirical mode decomposition and energy characteristics of discrete wavelet transform," Journal of Textile Research, vol. 39, 2018.

[9] C. Wang, A. Bernstein, J. Y. Le Boudec, and M. Paolone, "Explicit conditions on existence and uniqueness of load-flow solutions in distribution networks," IEEE Trans. Smart Grid, vol. 9, 2016.

[10] M. Imani, S. F. Ghoreishi, and U. M. Braga-Neto, "Bayesian control of large MDPs with unknown dynamics in data-poor environments," Advances in Neural Information Processing Systems, vol. 31, pp. 8157-8167, 2018.

[11] A. Bustos, H. Rubio, C. Castejón, and J. C. García-Prada, "EMD-based methodology for the identification of a highspeed train running in a gear operating state," Sensors, vol. 182, 2018.

[12] B. Gao, W. L. Woo, and S. S. Dlay, "Single channel blind source separation using EMD-subband VariableRegularized sparse features," IEEE Transactions on Audio Speech and Language Processing, vol. 19, 2011.

[13] A. Bin Queyam, S. Kumar Pahuja, and D. Singh, "Quantification of feto-maternal heart rate from abdominal ecg signal using empirical mode decomposition for heart rate variability analysis," Technologies, vol. 5, 2017.

[14] Y.-x. Li and L. Wang, "A novel noise reduction technique for underwater acoustic signals based on complete ensemble empirical mode decomposition with adaptive noise, minimum mean square variance criterion and least mean square adaptive filter," Defence Technology, vol. 16, 2019.

[15] Z. Wu and N. E. Huang, "Ensemble empirical mode decomposition: a noise-assisted data analysis method," Advances in Adaptive Data Analysis, vol. 1, 2009.

[16] J. Singh, A. K. Darpe, and S. P. Singh, "Bearing damage assessment using Jensen-Rényi Divergence based on EEMD," Mechanical Systems and Signal Processing, vol. 87, pp. 307-339, 2017.

[17] A. C. Marcelo and S. Gastón, "Improved complete ensemble EMD: a suitable tool for biomedical signal processing," Biomedical Signal Processing and Control, vol. 14, pp. 19-29, 2014.

[18] S. Tian, X. Bian, Z. Tang, K. Yang, and L. Li, "Fault diagnosis of gas pressure regulators based on CEEMDAN and feature clustering," IEEE Access, vol. 7, pp. 132492-132502, 2019.

[19] V. R. Carvalho, "Evaluating five different adaptive decomposition methods for EEG signal seizure detection and classification," Biomedical Signal Processing and Control, vol. 62, 2020 .

[20] L. El Bouny, M. Khalil, and A. Adib, "ECG signal filtering based on CEEMDAN with hybrid interval thresholding and higher order statistics to select relevant modes," Multimedia Tools and Applications, vol. 78, no. 10, pp. 13067-13089, 2019. 